\title{
Vortex generation in protoplanetary disks with an embedded giant planet
}

\author{
M. de Val-Borro ${ }^{1,2}$, P. Artymowicz ${ }^{3,2}$, G. D’ Angelo ${ }^{4}$, and A. Peplinski ${ }^{2}$ \\ 1 Harvard-Smithsonian Center for Astrophysics, 60 Garden St., Cambridge, MA 02138, USA \\ e-mail: mdeval@cfa.harvard.edu \\ 2 Stockholm University, AlbaNova University Center, 10691 Stockholm, Sweden \\ 3 University of Toronto at Scarborough, 1265 Military Trail, Toronto, Ontario M1C 1A4, Canada \\ ${ }^{4}$ NASA-ARC, Space Science and Astrobiology Division, MS 245-3, Moffett Field, CA 94035, USA
}

Received 25 January 2007 / Accepted 18 June 2007

\section{ABSTRACT}

\begin{abstract}
Context. Vortices in protoplanetary disks can capture solid particles and form planetary cores within shorter timescales than those involved in the standard core-accretion model.

Aims. We investigate vortex generation in thin unmagnetized protoplanetary disks with an embedded giant planet with planet to star mass ratio $10^{-4}$ and $10^{-3}$

Methods. Two-dimensional hydrodynamical simulations of a protoplanetary disk with a planet are performed using two different numerical methods. The results of the non-linear simulations are compared with a time-resolved modal analysis of the azimuthally averaged surface density profiles using linear perturbation theory.

Results. Finite-difference methods implemented in polar coordinates generate vortices moving along the gap created by Neptunemass to Jupiter-mass planets. The modal analysis shows that unstable modes are generated with growth rate of order $0.3 \Omega_{\mathrm{K}}$ for azimuthal numbers $m=4,5,6$, where $\Omega_{\mathrm{K}}$ is the local Keplerian frequency. Shock-capturing Cartesian-grid codes do not generate very much vorticity around a giant planet in a standard protoplanetary disk. Modal calculations confirm that the obtained radial profiles of density are less susceptible to the growth of linear modes on timescales of several hundreds of orbital periods. Navier-Stokes viscosity of the order $v=10^{-5}$ (in units of $a^{2} \Omega_{\mathrm{p}}$ ) is found to have a stabilizing effect and prevents the formation of vortices. This result holds at high resolution runs and using different types of boundary conditions.

Conclusions. Giant protoplanets of Neptune-mass to Jupiter-mass can excite the Rossby wave instability and generate vortices in thin disks. The presence of vortices in protoplanetary disks has implications for planet formation, orbital migration, and angular momentum transport in disks.
\end{abstract}

Key words. planet and satellites: general - accretion, accretion disks - hydrodynamics - instabilities - methods: numerical

\section{Introduction}

Stability of rotationally supported gas disks is an area of active research, motivated among other reasons, by a need to understand the origin and stability of hydrodynamics turbulence underlying the so-called anomalous viscosity in accretion disks. The concept of $\alpha$-turbulence in accretion disks was introduced more than three decades ago by Shakura \& Sunyaev (1973) to account for the angular momentum transfer and explain accretion onto the central object. The magnetorotational instability (MRI) has been proposed to explain the enhanced viscosity in hot and sufficiently ionized accretion disks with a Keplerian angular velocity profi le threaded by a weak magnetic fi eld (Balbus \& Hawley 1991; Balbus et al. 1996; Balbus \& Hawley 1998). However, in the context of cold protoplanetary disks, the ionization by cosmic rays and stellar radiation is limited to the surface layers of the disk while the so called "dead zone" in the vicinity of the central plane is expected to have low ionization (Gammie 1996). In some astrophysical systems such as cataclysmic variables and outer regions of active galactic nuclei the coupling between the magnetic field and the gas is also weak and MHD effects may be negligible.
The stability of differentially rotating disks has been considered analytically and numerically in the purely hydrodynamical case (Papaloizou \& Pringle 1984, 1985; Goldreich et al. 1986; Papaloizou \& Pringle 1987) with applications to circumstellar disks and galactic disks. A rotating isentropic torus with a gradient of specific angular momentum is found to be unstable to low-order non-axisymmetric perturbations due to the Papaloizou-Pringle instability. Several mechanisms have been proposed that are able to sustain purely hydrodynamical turbulence and generate an anomalous $\alpha$-viscosity in accretion disks (Li et al. 2000; Klahr \& Bodenheimer 2003; Mukhopadhyay et al. 2005). Dubrulle et al. (2005) studied non-axisymmetric instabilities in stratifi ed Keplerian disks using numerical and analytical methods. A linear instability appears for Reynolds numbers of order $10^{3}$ and perturbations with characteristic scales smaller than the vertical scale of the disk, assuming the angular velocity decreases with radius. These results suggest that despite the stabilizing effect of the Coriolis force, a Keplerian flow may undergo a transition to turbulence. Nevertheless, some of those mechanisms may depend on boundary or edge effects.

Rossby waves in thin Keplerian disks have been studied in the linear approximation (Lovelace et al. 1999; Li et al. 2000) and with fully non-linear numerical simulations (Tagger 2001). 
The existence of unstable modes has been found to be associated with radial gradients of an entropy-modifi ed version of vortensity. Rossby waves in disks break up forming vortices in the nonlinear limit ( $\mathrm{Li}$ et al. 2001) in agreement with the predictions from linear theory. The dispersion relation of this Rossby Wave Instability (RWI) is analogous to the one for Rossby waves in planetary atmospheres. Reynolds stresses produced by the RWI can yield outward transport of angular momentum and accretion onto the central star. Varnière \& Tagger (2006) have discussed the generation of Rossby waves in the "dead zones" of protoplanetary disks where they may enhance the accretion rate of solids and favor planet formation. The angular momentum transport in disks around supermassive black holes at the center of galaxies can also be explained by the formation of Rossby vortices when there is a steep enough density gradient (Colgate et al. 2003). The angular momentum transfer due to vortices in galactic disks is found to be greater than in an $\alpha$-viscosity disk. Recently, Tagger \& Melia (2006) have described a magnetohydrodynamics version of the RWI and applied it to the study of the quasiperiodic oscillations in Sgr A*. Rossby waves can also appear in thin planetary atmospheres with solid rotation leading to the formation of vortices like Jupiter's "Great red spot" (Marcus 1988).

Long-lived vortices are able to capture solid materials to form massive bodies and speed up the formation of planetary cores (Barge \& Sommeria 1995; Bracco et al. 1999; Klahr $\&$ Bodenheimer 2006). The stability of three-dimensional vortices in a three dimensional stratifi ed disk has been studied by Barranco \& Marcus $(2005,2006)$ using spectral anelastic hydrodynamics simulations. They fi nd that vortices are hydrodynamically stable for several orbits away from the mid-plane of the disk. The formation of vortices in the corotation region excited by a protoplanet has been studied numerically by Balmforth \& Korycansky (2001) including the saturation of the corotation torque and the effects of dissipation in the non-linear dynamics of the flow.

Several numerical schemes studied by de Val-Borro et al. (2006) show vortex formation but the aim of the study did not include a detailed investigation of vortex generation. In this paper, we intend to look at this formation process in more detail. We also wish to check that codes that do not predict vortex generation do not artifi cially damp unstable modes due to the numerical viscosity. Some simulations produce waves and vortices at the edge of the gap, which could in principle interact with the wake to cause semi-periodic disturbances propagating away along the shock. In simulations using the Piecewise Parabolic Method (hereafter PPM), low- $m$ perturbations are observed at the edges of the gap (Ciecielag et al. 2000). Wave-like disturbances with mode number $\sim 5$ are observed at the edge of the gap created by a Jupiter-size planet in the numerical results presented by Nelson \& Benz (2003). Instabilities close to the planet or along the edges of the gap created by a giant planet, as well as the time variability of the flow near the Roche lobe may affect the speed and direction of planetary migration.

In this paper, we study the effect of an annular gap cleared by a planet on the stability of a protoplanetary disk. We consider non-axisymmetric linear perturbations to the inviscid and compressible Euler equations. In Sect. 2, we present the semianalytical methods used to study the stability of disks. We describe the numerical codes in Sect. 3. In Sect. 4 we present the results of the numerical simulations and the perturbative linear analysis. We discuss the results in the context of protoplanetary disks in Sect. 5. Finally, the numerical diffusivity in our numerical codes is calibrated in Appendix A.

\section{Modal analysis}

We perform a modal analysis of analytical and numerically obtained density profi les, in order to see if there is agreement between the vortex generation in the simulations and growing unstable modes in the linear stability analysis. Linear perturbative analysis provides a valuable tool to study the stability of disks. The solution of the linearized Euler equations is treated along the lines of the work of Lovelace et al. (1999) and Li et al. (2000), where the stability can be evaluated solving a numerical eigenvalue problem for a given profi le. Alternatively, the growth of the initial perturbations can be determined by solving the equations as an initial value problem.

We consider non-axisymmetric small perturbations sinusoidally varying in azimuth to the inviscid Euler equations

$\frac{\partial \tilde{\Sigma}}{\partial t}+\nabla \cdot(\tilde{\Sigma} \tilde{\boldsymbol{v}})=0$

$\frac{\partial \tilde{\boldsymbol{v}}}{\partial t}+(\tilde{\boldsymbol{v}} \cdot \nabla) \tilde{\boldsymbol{v}}=-\frac{1}{\tilde{\Sigma}} \nabla \tilde{P}-\nabla \Phi$

where $\tilde{\Sigma}$ is the surface mass density, $\tilde{P}$ the vertically integrated pressure, $\tilde{\boldsymbol{v}}$ the velocity of the flow and $\Phi$ is the gravitational potential. The perturbed quantities have the form $\tilde{\Sigma}=\Sigma+\delta \Sigma(r, \phi, t)$, $\tilde{P}=P+\delta P(r, \phi, t)$ and $\tilde{\boldsymbol{v}}=\boldsymbol{v}+\delta \boldsymbol{v}(r, \phi, t)$ with perturbations in the disk plane depending on the azimuthal angle as $\propto \exp [\mathrm{i}(m \phi-\omega t)]$, where $m$ is the azimuthal mode number and $\omega=\omega_{r}+\mathrm{i} \gamma$ is the complex mode frequency. The initial velocity component in the radial direction is neglected and the angular velocity $\Omega$ is obtained from the force balance between gravity, pressure gradients and centrifugal force

$\Omega^{2}=\frac{1}{r}\left[\frac{1}{\Sigma} \frac{\mathrm{d} P}{\mathrm{~d} r}+\frac{\mathrm{d} \Phi}{\mathrm{d} r}\right]$

we use a barotropic equation of state, $P \propto \Sigma^{\Gamma}$ with adiabatic index $\Gamma=5 / 3$, to perform the modal analysis on the analytical disk profi les studied by Lovelace et al. (1999).

A locally isothermal equations of state is used to calculate unstable modes from the numerical simulations described in Sect. 3 . The vertically integrated pressure is given by

$P=c_{\mathrm{s}}^{2} \Sigma$

where $c_{\mathrm{s}}$ is the sound speed. The temperature can be calculated from the ideal gas equation of state

$P=\frac{\mathcal{R} \Sigma T}{\mu}$,

where $\mathcal{R}$ is the gas constant and $\mu$ is the mean atomic weight. The initial unperturbed density is uniform in our simulations and the temperature has a fi xed profi le $\propto \bar{r}^{1}$ for the isothermal calculations, where $r$ is the distance from the rotation axis. The sound speed profi le of the disk is that of a standard slightly flaring solar nebula with constant aspect ratio

$c_{\mathrm{s}}=0.05 \sqrt{\frac{G M_{*}}{r}}$

where $G$ is the gravitational constant and $M_{*}$ the mass of the central star.

The linearized equations can be reduced to a second order differential equation for the enthalpy of the fluid, $\eta=\delta P / \Sigma$, in the general case when the pressure is a function of both 
density and temperature (Lovelace et al. 1999; Li et al. 2000; de Val Borro \& Artymowicz 2004).

$\eta^{\prime \prime}+B(r) \eta^{\prime}+C(r) \eta=0$

where the coefficients $B(r)$ and $C(r)$ depend on the radial distance,

$$
\begin{aligned}
B(r)= & \frac{1}{r}+\frac{\mathcal{F}^{\prime}}{\mathcal{F}}-\frac{\Omega^{\prime}}{\Omega} \\
C(r)= & -k_{\phi}^{2}-\frac{\kappa^{2}-\Delta \omega^{2}}{c_{\mathrm{s}}^{2}}-2 k_{\phi} \frac{\Omega}{\Delta \omega} \frac{\mathcal{F}^{\prime}}{\mathcal{F}} \\
& -\frac{1-L_{\mathrm{s}}^{\prime}}{L_{\mathrm{s}}^{2}}-\frac{B(r)+4 k_{\phi} \Omega / \Delta \omega}{L_{\mathrm{s}}}-\frac{k_{\phi}^{2} c_{\mathrm{s}}^{2} / \Delta \omega^{2}-1}{L_{\mathrm{s}} L_{\mathrm{p}}}
\end{aligned}
$$

and

$$
\mathcal{F}(r)=\frac{\Sigma \Omega}{\kappa^{2}-\Delta \omega^{2}-c_{\mathrm{s}}^{2} /\left(L_{\mathrm{s}} L_{\mathrm{p}}\right)}
$$

$k_{\phi}=\frac{m}{r}$

$\kappa^{2}=\frac{1}{r^{3}} \frac{\mathrm{d} l^{2}}{\mathrm{~d} r}$

$\Delta \omega(r)=\omega_{r}+i \gamma-m \Omega(r)$

where $\kappa$ is the epicyclic frequency, $l=\Omega r^{2}$ is the angular momentum per unit mass, $c_{\mathrm{s}}$ the adiabatic sound speed and $L_{\mathrm{s}}, L_{\mathrm{p}}$ are the equilibrium length scales of variation of entropy and pressure changes, given by:

$L_{\mathrm{s}}^{-1}=\frac{1}{\Gamma} \frac{\mathrm{d} \ln \left(P / \Sigma^{\Gamma}\right)}{\mathrm{d} r}$

$L_{\mathrm{p}}^{-1}=\frac{1}{\Gamma} \frac{\mathrm{d} \ln (P)}{\mathrm{d} r}$.

$L_{\mathrm{s}}$ and $L_{\mathrm{p}}$ are calculated numerically from the averaged profi les obtained in the simulations using Eq. (4).

The growth of the unstable modes can form vortices or Rossby waves in the nonlinear regime ( $\mathrm{Li}$ et al. 2001). The Rayleigh criterion states that the disk will be stable to axisymmetric perturbations when the specifi $c$ angular momentum increases with radial distance. For a Keplerian disk the epicyclic frequency is always positive, $\kappa^{2}=\Omega_{\mathrm{K}}^{2}$, where the Keplerian angular frequency is given by $\Omega_{\mathrm{K}}=\left(G M_{*} / r^{3}\right)^{1 / 2}$, and therefore axisymmetric waves will be stable. However, when the pressure effects are taken into account it is possible to have axisymmetric instabilities for a sufficiently large pressure gradient according to the Solberg-Høiland criterion,

$\kappa^{2}(r)+N_{r}^{2}(r) \geq 0$

where

$N_{r}^{2}(r)=-\frac{1}{\Gamma \Sigma} \frac{\mathrm{d} P}{\mathrm{~d} r} \frac{\mathrm{d}}{\mathrm{d} r} \ln \left(\frac{P}{\Sigma^{\Gamma}}\right)$

is the square of the Brunt-Väisälä frequency in the radial direction.

The eigenproblem for the perturbed enthalpy is solved using two semi-analytical methods. One way of fi nding the complex eigenfrequency is the shooting method, where the integration proceeds from the disk boundaries to an intermediate fi tting point, where continuity of the eigenfunction and its fi rst derivative is required. Our implementation uses a leapfrog method to integrate the equation from the boundaries to the fi tting point. The values of the entalphy and its derivative at the starting points are specifi ed based on several prescriptions using outgoing spiral waves (Li et al. 2000) and vanishing eigenfuntion at the boundaries. We fi nd that the obtained growth rates do not depend sensitively on the choice of boundary conditions. Several root fi nding algorithms in the complex plane can be used to fi nd the unstable modes. The winding number theorem uses closed-path integrals (e.g. Kargl \& Marston 1989) to fi nd the number of roots inside a closed contour.

The winding number theorem states that for a complex analytic function $f(\omega)$ defi ned inside a contour $C$

$2 \pi \mathrm{i}(N-P)=\oint \frac{f^{\prime}(\omega)}{f(\omega)} \mathrm{d} \omega$

where $N$ is the number of roots and $P$ is the number of poles inside the contour $C$, and the integral is defi ned in counterclockwise sense. We look for contours that comprise a single root and are not located close to solutions or singularities on the real axis. There are singularities in Eq. (7) at the corotation resonance $\Delta \omega=0$ and when a modifi ed form of the Lindblad resonance for non-barotropic flow is satisfi ed, $\kappa^{2}-\Delta \omega^{2}-c_{\mathrm{s}}^{2} /\left(L_{\mathrm{s}} L_{\mathrm{p}}\right)=0$. It is important to avoid branch points close to the contours since the winding number method would not give the right number of roots. In the case of analytical density profi les given by hyperbolic trigonometric functions the branch points do not affect the contours used to fi nd solutions with positive growth rate.

The solutions are then calculated by

$$
\sum_{i=1}^{N} \zeta_{i}-\sum_{j=1}^{M} \xi_{j}=\frac{1}{2 \pi i} \oint \frac{\omega f^{\prime}(\omega)}{f(\omega)} \mathrm{d} \omega
$$

where $\zeta_{i}$ are the zeros and $\xi_{j}$ the poles of the complex function $f(\omega)$. The mode frequency can be determined in a contour with a single root. A multidimensional Newton-Raphson method (Press et al. 1992) is then employed to locate the roots with arbitrary accuracy.

Another approach to solve Eq. (7) involves discretizing the equation on a fi nite grid and use appropriate boundary conditions to reduce the problem to fi nding numerically the roots of the determinant of a complex tridiagonal matrix (e.g., Laughlin et al. 1998; Li et al. 2000). We solved the determinant using the previous root fi nding algorithm and obtain the radial profi le for the eigenfunction $\eta(r)$ and the perturbed variables.

We checked these two methods on the axisymmetric analytical step jump profi les in surface density studied by Li et al. (2000). We considered azimuthal mode numbers from $m=1$ to 10 and calculated the growth rates of the unstable modes and the corresponding eigenfunctions. For analytical density profi les with various shapes both our methods agree with the results of $\mathrm{Li}$ et al. (2000). We tested the dependence of the solution on the shape of the pressure profi le and aspect ratio of the disk. The eigenfunction for density profi les with a locally isothermal equation of state is obtained by solving the discretized Eq. (7).

In Fig. 1, we show the real part of the mode frequency as a function of the azimuthal number for the averaged density profi les of a NIRVANA simulation (see Sect. 3) at $n_{\mathrm{r}} \times n_{\phi}=$ $256 \times 768$ resolution after 10 orbits. Figure 2 shows the growth rate as a function of the mode number for the same averaged density profi le, after 10 orbital periods when the threshold for the excitation of the instability is reached. The density slope in 


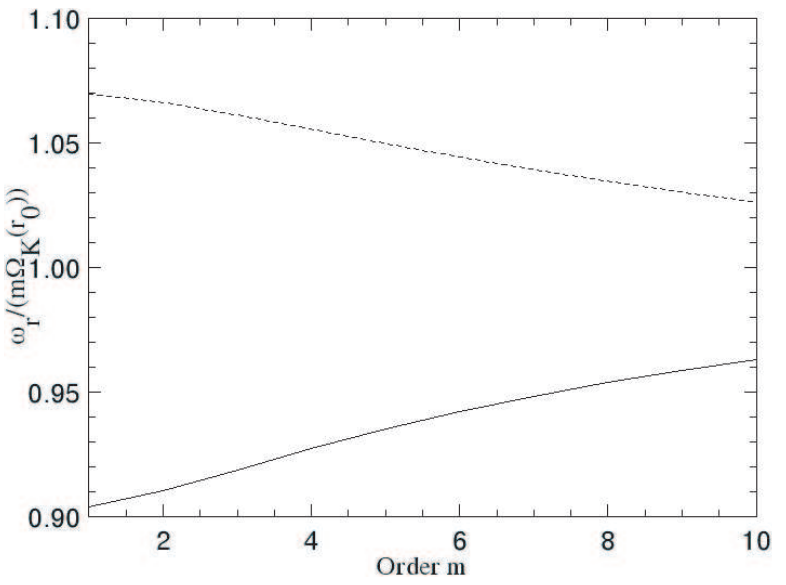

Fig. 1. Real frequency of the most unstable modes for a gap opened by a Jupiter-mass planet after 10 periods as a function of the azimuthal mode number. The solid line shows the mode frequency at the outer edge of the gap and the dashed line is the mode frequency at the inner edge divided by $m \Omega_{\mathrm{K}}\left(r_{0}\right)$ where $\Omega_{\mathrm{K}}\left(r_{0}\right)$ is the Keplerian angular frequency at the planet position.

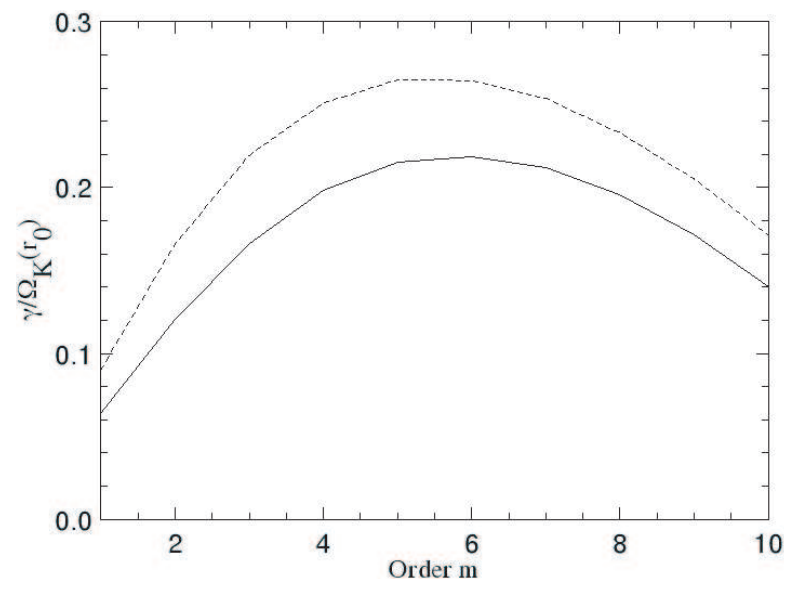

Fig. 2. Growth rate for a gap opened by a Jupiter-mass planet after 10 orbits against the azimuthal mode number. The solid line shows the growth rate at the outer edge of the gap and the dashed line is the growth rate at the inner edge. The growth rate of the instability peaks at mode numbers 5-6.

the simulations is described using two parameters. The depth of the gap is calculated with respect to the unperturbed density in the inner and outer disk. The length scale over which the density varies is estimated to be the difference between the local maximum and minimum at both inner and outer disks. An analytical jump function is fi tted to the averaged profi les.

The local maximum at the planet position in the averaged profi les is not expected to change the growth rates signifi cantly. In addition, most of the gap is clean, apart from the material close to the planet position and at Lagrangian points $L_{4}$, and $L_{5}$. In the simulations with an initial gap the averaged profi les do not have local maxima inside the gap after 100 orbits.

The larger growth rates in Fig. 2 correspond to the more unstable modes of Eq. (7) that will dominate the solution. The inner edge of the gap opened by a planet, shown by the dashed line, has higher growth rate than that of the outer edge, represented by the solid line, at a given time. This difference can be due to the fact that the inner boundary is closer to the location of the instability in the inner disk than it is the outer boundary to the corresponding instability outside the planet's radius. The real frequency of
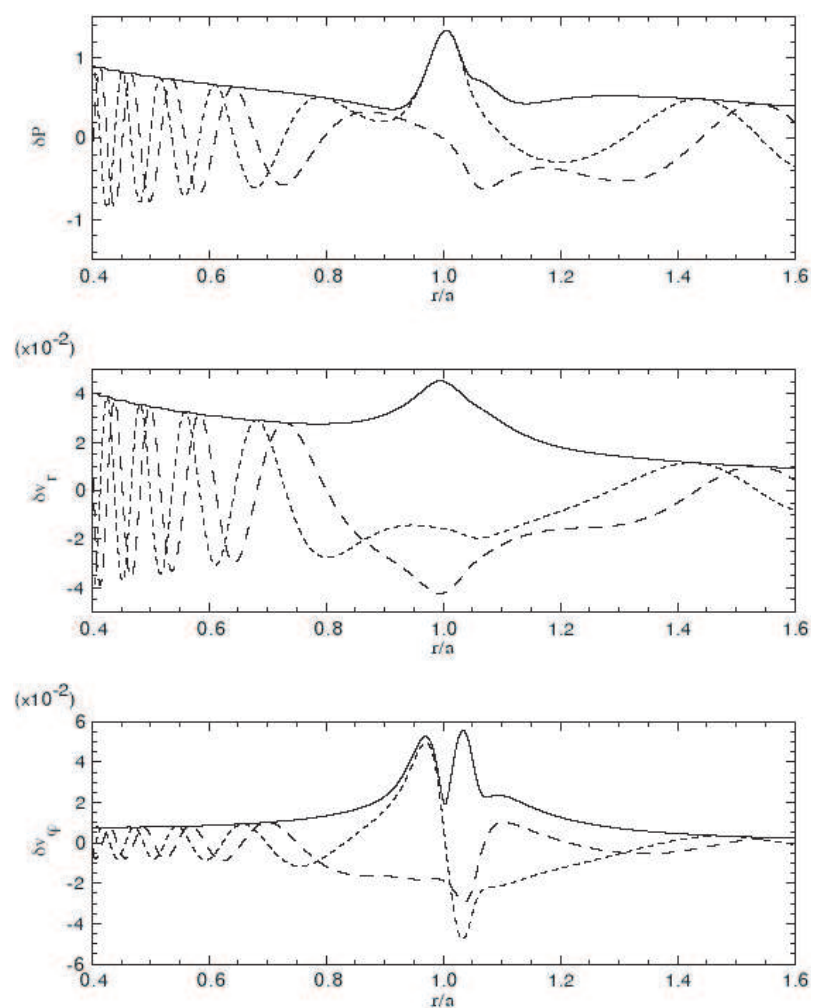

Fig. 3. Radial profile of the eigenfunctions for the outer edge of the gap at $t=10$ periods and mode number $m=5$. From top to bottom the pressure perturbation and radial and azimuthal perturbed velocity components are shown. The dotted and dashed lines are the real and imaginary part of the eigenfunctions. The amplitude is shown by the solid line which peaks at the position of the edge for the eigenfunction of the perturbed pressure.

the modes correspond to a radial location just outside the corotating region where vortices are formed in the simulations after about 10 orbits. The number of vortices in the numerical results is consistent with the growth rates peaking at $m=4-6$.

In Fig. 3 the real and imaginary parts of the radial eigenfunction of the perturbed variables at the outer edge of the gap are plotted for azimuthal mode $m=5$. The eigenfunction corresponds to a time when the gap becomes deep enough to generate modes with positive growth rate. The middle and bottom panel show the eigenfunctions of the perturbations of the velocity components. The radial eigenfunctions at the inner edge of the gap after 10 orbits for azimuthal mode $m=5$ are plotted in Fig. 4 .

\section{Numerical codes}

We performed 2-dimensional hydrodynamical simulations using two independent grid-based codes, implemented in cylindrical and Cartesian coordinates. The simulations were run on a uniform grid for 100 orbital periods Different boundary conditions were tested to avoid reflection of waves at the boundaries.

\subsection{Initial setup}

The computations were performed in the radial domain $0.4 a \leq$ $r \leq 2.5 a$ where $a$ is the planet semi-major axis. The disk is 


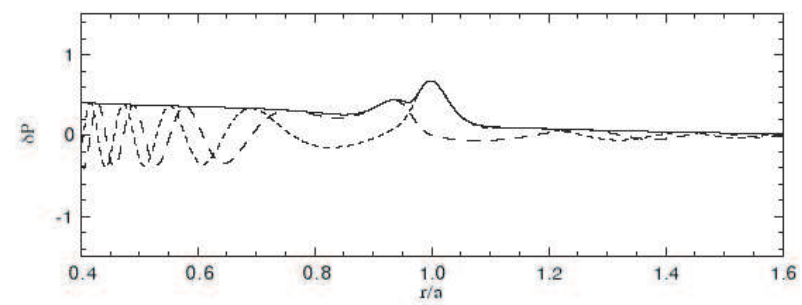

$\left(\times 10^{-2}\right)$
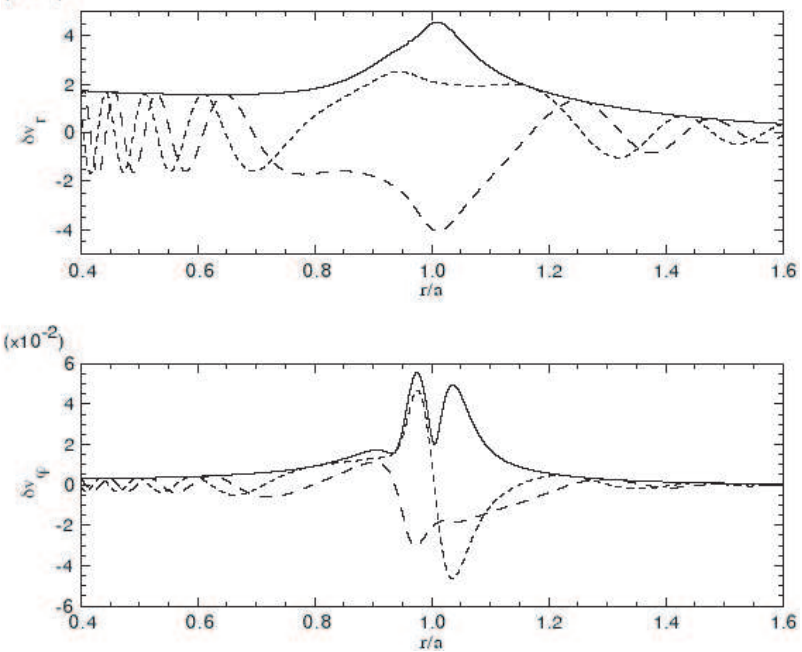

Fig. 4. Radial profile of the eigenfunctions for the inner edge of the gap at $t=10$ periods and mode number $m=5$. From top to bottom the pressure perturbation and radial and azimuthal perturbed velocity components are shown. The dotted and dashed lines are the real and imaginary part of the eigenfunctions.

assumed to be geometrically thin and the fluid equations are solved using vertically-integrated variables:

$\Sigma(r, \phi)=\int_{-H}^{H} \rho(r, \phi, z) \mathrm{d} z, \quad P(r, \phi)=\int_{-H}^{H} p(r, \phi, z) \mathrm{d} z$

where $H$ is the vertical scale height.

The planet's gravitational potential was given by the formula

$\phi=\frac{-\mu}{\sqrt{s^{2}+\epsilon^{2}}}$

where $\epsilon$ is the gravitational softening and $s$ is the distance from the planet. The softening is defi ned as $\epsilon=0.6 H_{\mathrm{p}}$, with $H_{\mathrm{p}}$ the disc scale height at the planet location. This softening was introduced to reproduce the torque cut-off due to 3-dimensional effects at distance from the planet larger than $H_{\mathrm{p}}$. The softening length is comparable in size to the characteristic size of the Roche lobe (along the planet's orbit) and thus it is likely to affect the flow dynamics in the corotation region but it is unlikely to strongly affect the dynamics in the regions where we observe vortex formation. Initially, the gas rotates with Keplerian angular frequency around the central star. The mass ratio had the values $\mu=M_{\mathrm{p}} / M_{*}=10^{-3}$ and $10^{-4}$, corresponding to Jupiter and Neptune masses when the stellar mass is one solar mass. Self-gravity of the disk was not included in the simulations. The planet was kept in a coplanar, circular orbit at $a=1$. We did not consider the accretion of disk material onto the protoplanet.

We assume that the disk radiates efficiently the thermal energy generated from tidal dissipation and viscous heating. In the absence of a radiation mechanism the gas would heat up and the disk could become geometrically thick. In our models, we use a locally isothermal equations of state, (Lin \& Papaloizou 1985) with constant aspect ratio $H / r=0.05$.

The vortensity or potential vorticity is calculated in the corotating frame:

$\zeta=\left(\nabla \times v+2 \Omega_{\mathrm{p}}\right) / \Sigma$

where $\Omega_{p}$ is the orbital frequency of the planet.

We perform our simulations in the inviscid limit. In addition, a few cases with physical viscosity $v=10^{-6}$ and $10^{-5} a^{2} \Omega_{\mathrm{p}}$ are considered in order to estimate a threshold for the formation of vortices. Artifi cial viscosity is not needed to smooth the shocks in our codes, which is important to study the formation of vortices. The codes are also able to resolve the large density contrast between the corotating region and the rest of the disk. Diffusion into the gap opened by the planet may result from numerical viscosity or shocks. The numerical viscosity in our codes is estimated in Appendix A for inviscid runs without tidal perturbations. These tests confi rm that numerical diffusion is not affecting our results.

The initial density was uniform and the gravity of the planet was introduced gradually with the formula

$M(t)=M_{\mathrm{p}} \sin ^{2}\left(\frac{\pi t}{10 P_{\mathrm{p}}}\right)$

we have used timescales between 5-10 orbital periods to introduce the gravity from the planet. Although the time when the instability appears depend on how the gravity is started, there is agreement between the modal analysis and numerical simulations for timescales of 5 and 10 orbits. The results presented in Sect. 4 use a switch-on time of the gravity of 5 orbits.

In some of the calculations we use an initial gap profi le derived under the WKB approximation (e.g., Lubow \& D' Angelo 2006)

$\Sigma_{0}(r)=\exp \left[-\frac{f}{9} \frac{q^{2} a^{2} \Omega_{\mathrm{p}}}{v_{\mathrm{p}}}\left(\frac{a}{\Delta_{\mathrm{p}}}\right)^{3}\right]$,

where we use $f / \nu_{\mathrm{p}}$ between $1.7 \times 10^{4}$ and $1.8 \times 10^{4}$ and $\Delta_{\mathrm{p}}$ is the maximum of $H$ and $|r-a|$. This gap profi le allows us to check whether a rapid formation of the gap is necessary for the presence of instabilities.

The Cartesian implementation of FLASH was run on a uniform non-rotating grid at resolution $n_{x} \times n_{y}=320 \times 320$, and $n_{x} \times n_{y}=640 \times 640$. The computational domain was $-2.6 a \leq$ $x \leq 2.6 a$ and $-2.6 a \leq y \leq 2.6 a$.

For computational convenience the unit of time used in the simulations was the orbital period at the planet location, $P_{\mathrm{p}}=$ $2 \pi\left[a^{3} / G\left(M_{*}+M_{\mathrm{p}}\right)\right]^{1 / 2}=2 \pi$, where $G\left(M_{*}+M_{\mathrm{p}}\right)=1$ and $a=1$. Therefore, the angular frequency of the planet was $\Omega_{\mathrm{p}}=1$ in our units.

We used solid boundaries with wave damping zones next to the boundaries to avoid wave reflection that can create artifi cial resonances. The damping regions were implemented in the NIRVANA simulations at $0.4 a \leq r \leq 0.5 a$ and $2.1 a \leq r \leq 2.5 a$, by solving the following equation after each timestep:

$\frac{\mathrm{d} x}{\mathrm{~d} t}=-\frac{x-x_{0}}{\tau} R(r)$

where $x$ represents the surface density and velocity components, $\tau$ is the orbital period at the corresponding boundary, and $R(r)$ is a parabolic function which is one at the domain boundary and zero at the interior boundary of the wave killing regions. NIRVANA simulations were also run using alternative boundary conditions to check that the gap profi les and vorticity in the corotating region do not depend strongly on boundary conditions. 


\subsection{NIRVANA}

Our NIRVANA implementation is based on the original version of the code by Ziegler \& Yorke (1997). The Navier-Stokes equations are solved using a directional operator splitting upwind scheme which is second-order accurate in space and semisecond-order in time.

NIRVANA uses a staggered grid where scalar quantities are stored at the center of grid cells and vectors are stored at the cell boundaries. The frame is centered on the center of mass of the star-planet system and rotates with the same angular frequency as the planet orbits the central star. The code was run with both non-reflective boundary conditions described above (see also Godon 1996) and wave-killing zones close to the boundaries (de Val-Borro et al. 2006). These two types of boundary conditions provide consistent results since in both cases wave reflection in the boundaries is reduced. A Courant number of 0.5 was used in the simulations.

Two grid resolutions were considered in which the number of cells in the radial and azimuthal directions were $n_{\mathrm{r}} \times n_{\phi}=$ $256 \times 768$ and $512 \times 1536$, respectively, with uniform spacing in both dimensions. Therefore, the cells around the planet position were approximately square.

\subsection{FLASH}

The FLASH code (Fryxell et al. 2000) is a fully parallel AMR implementation of the PPM algorithm in its original Eulerian form ${ }^{1}$ (Woodward \& Colella 1984; Colella \& Woodward 1984). FLASH has been extensively tested in various compressible flow problems with astrophysical applications (Calder et al. 2002; Weirs et al. 2005).

Our implementations of the FLASH code used both polar coordinates and the original Cartesian formulation of FLASH. The polar version of FLASH was run in the corotating coordinate system while the Cartesian implementation was run in the inertial frame. Our implementations were based on release 2.5 of FLASH with customized modules for the equation of state and gravity forces, explicitly ensuring the conservative transport of angular momentum in the angular sweep. This is particularly important when large density gradients are present in the disk. The Coriolis forces were treated conservatively as described by Kley (1998). The isothermal Riemann solver was ported from the AMRA code (Plewa \& Müller 2001). Courant numbers of 0.7 and 0.8 were used in the simulations.

The Cartesian grid was centered in the center of mass of the system and the orbits of the planet and the central star were integrated using a simple Runge-Kutta method. The grid cells were sized to give the same radial resolution in both implementations, although since the grid went to $r=0$ in the Cartesian simulations, the grid size had to be larger to achieve the same resolution. The Cartesian code was run at resolutions $n_{x} \times n_{y}=320 \times 320$ and $640 \times 640$. To improve the angular resolution close to the central star, an additional level of refi nement was used in the inner disk in the Cartesian implementation. Different timesteps were used for each level of refi nement to speed up the simulation.

The damping condition described in de Val-Borro et al. (2006) was applied in the ring $2.1 a \leq r \leq 2.5 a$ close to the outer boundary but not in the inner disk for the Cartesian FLASH implementation. A free outflowing boundary was used at the outer

\footnotetext{
${ }^{1}$ FLASH is available at http://www. flash.uchicago.edu/
}

boundaries $x= \pm 2.6 a$ and $y= \pm 2.6 a$. and there was free gas flow inside $0.4 a$.

The cylindrical implementation used a frame centered on the star with resolutions $n_{r} \times n_{\phi}=256 \times 768$ and $512 \times 1536$ and wave-damping zones close to the inner and outer boundaries. The Coriolis force, centrifugal force, and indirect terms due to the fact that the center of the frame is displaced from the center of mass of the system are included in the equation of motion.

\section{Results}

We carried out modal growth analysis on the density profi les of protoplanetary disks perturbed by an embedded giant planet ranging from a Neptune to a Jupiter mass. The time resolved linear analysis was performed on the azimuthally averaged density profi les obtained from calculations, using a locally isothermal equation of state. In the following description, we will show the results of inviscid runs at different resolutions with an embedded Jupiter-mass protoplanet using different boundary conditions. Results from runs with physical viscosity will also be presented.

The real frequency and growth rate of the most unstable modes, as a function of time, are plotted in Fig. 5 for NIRVANA and FLASH polar simulations with resolution $n_{r} \times n_{\phi}=256 \times 768$ and wave-damping boundary conditions. The threshold for the appearance of the instability occurs after about 5 periods when the gap is sufficiently deep and the growth rate becomes positive. The edge of the gap in the simulations becomes nonaxisymmetric at this time and depressions in vortensity appear along the gap with $m=5-6$ symmetry which coincide with the azimuthal number of the most unstable modes shown in Fig. 2. Small vortices are observed in the inner and outer disk shortly afterwards with the same angular distribution.

In the bottom panel of Fig. 5, the growth rate from the inner disk edge, represented by crosses and circles for the different models, is larger than the growth rate at the outer disk, represented by dots and stars. This difference may be artifi cial because the inner boundary of the grid is closer to the planet location that the outer boundary is. The mode frequencies and growth rates for NIRVANA and polar FLASH agree within $10 \%$. This is in agreement with the vortex sizes obtained from the Fourier analysis of the gravitational torques on the planet. In the end of the simulation, the growth rate of the instability is a fraction of the angular velocity at the edge of the gap. The disk becomes unstable to axisymmetric perturbations at time $\sim 40$ orbits for the Jupiter simulations according to the Rayleigh criterion (Eq. (17)). The RWI grows exponentially in agreement with the linear analysis during the fi rst orbits (Li et al. 2001) and produces vortices that can be sustained by interaction with the spiral arms generated by the planet.

The linear analysis of Cartesian FLASH averaged profi les shows the presence of unstable modes in the inner and outer disk with growth rates of $\sim 0.2 \Omega_{\mathrm{K}}\left(r_{0}\right)$. In some Cartesian FLASH runs there are mode solutions with large growth rates that appear at late times, when the gap is becoming deeper. At the same time there are indications that some mild instability is happening in the disk. However, these are not the fast-growing modes found in the polar grid calculations with azimuthal number $m=4-6$. We do not fi nd stationary solutions with positive growth rates that remain for a time of order of the growth timescale in the Cartesian grid models. This is consistent with the fact that no vortices appear in the edges of the gap in these simulations after tens of orbital periods, as shown in Fig. 9. 

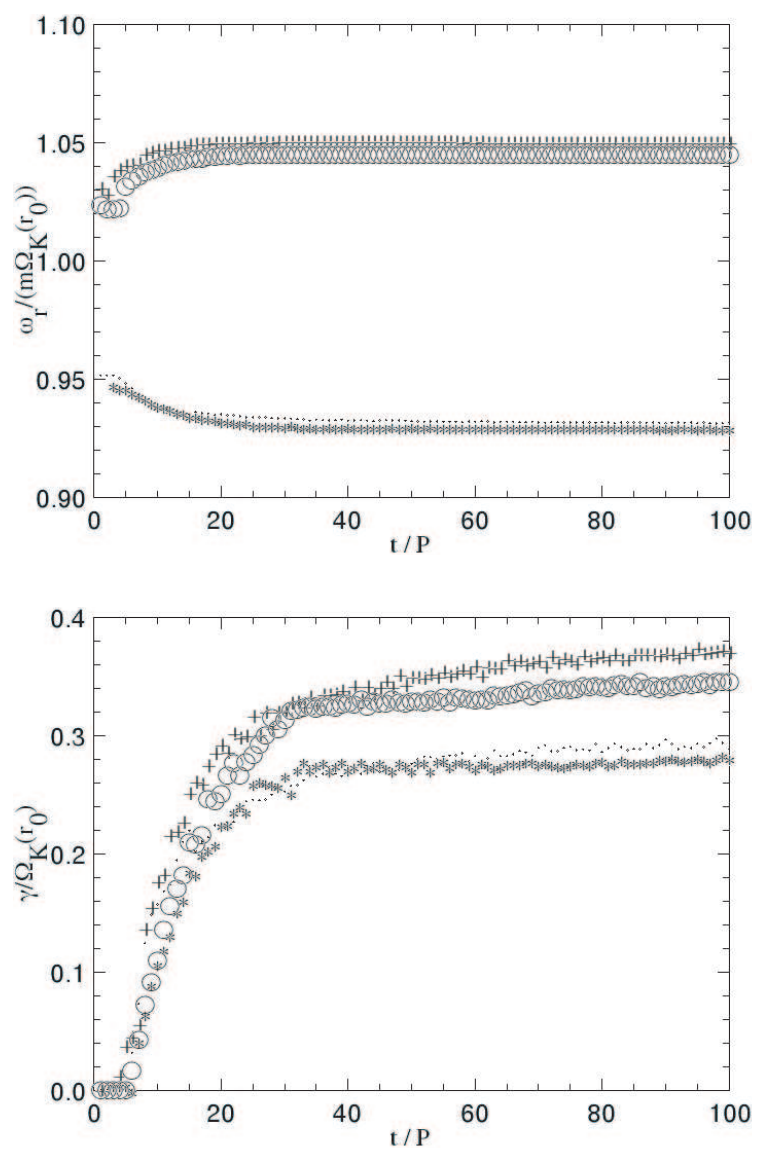

Fig. 5. Real frequency and growth rates of the unstable modes with azimuthal number $m=5$, as a function of time, for NIRVANA and polar FLASH simulations with resolution $n_{r} \times n_{\phi}=256 \times 768$. In the top panel, the crosses represent the mode frequencies at the outer edge of the gap and the dots are the mode frequencies at the inner edge for NIRVANA. The circles are the mode frequencies at the outer edge of the gap and the stars are the mode frequencies at the inner edge for polar FLASH. The frequencies are divided by $m \Omega_{\mathrm{K}}\left(r_{0}\right)$, where $\Omega_{\mathrm{K}}$ is the Keplerian angular frequency and $r_{0}$ is the radius at the edge of the gap. The crosses in the bottom panel are the growth rates at the outer gap edge and the dots are the growth rates at the inner edge for a NIRVANA calculation. The circles are the growth rates at the outer edge of the gap and the stars are the growth rates at the inner edge for a polar FLASH calculation. Growth rates are divided by the Keplerian frequency at the edge of the gap.

Figure 6 shows the density distribution for Jupiter inviscid simulations in logarithmic scale using different boundary conditions for NIRVANA and FLASH models. The left panels show the density contours at $t=50$ orbital periods and the right panels after $t=100$ orbits. There are two vortices moving along the outer edge with different phase velocities in all the simulations at $t=50$ periods. Those vortices will merge and form a large vortex at a later time producing strong oscillations on the torque exerted on the planet.

In Fig. 7 we show the growth rates with azimuthal number $m=5$ for the outer edge of the gap using an initial density with a gap given by Eq. (25). The gap shape tends to a steady state towards the end of the simulation. The growth rates agree within $5 \%$ (by the end of the simulation) with the results obtained using an initial uniform density.

Figure 8 shows the profi les averaged over the azimuth for the NIRVANA simulations using various boundary conditions. The slope of the gap is steeper at the inner edge than at the outer edge for the cylindrical schemes. The density peak in the inner disk is about $50 \%$ of the peak at the outer gap edge. The overall jump in density is larger at the inner edge despite the fact that the gap is slightly deeper just outside the planet's orbit. This produces a larger growth rate inside the corotation region (see Fig. 2). In the cylindrical FLASH simulation, the mass loss at the boundaries is greater but this does not affect the results of the modal calculation. The Cartesian FLASH results have a deeper gap and smaller density peaks at the edge of the gap. However, the growth rate of their most unstable modes is about $50 \%$ compared with that provided by the polar FLASH simulations.

The size of the peaks in the power spectrum of the torques on a Jupiter-mass planet, from different regions in the disks, are shown in Tables 1 and 2 . The torques are calculated excluding the material inside the Roche lobe of the planet, where the resolution may not be good enough to resolve the circumplanetary disk. NIRVANA calculations have a larger peak, which correspond to the amplitude of the vortices moving along the edge of the gap. This is consistent with larger vortices being observed in the density distributions of NIRVANA calculations in Fig. 6. FLASH calculations have PDS peaks which are between one and two orders of magnitude smaller. The frequencies in the corotating frame are close to the local Keplerian angular frequency at the edges of the gap. The difference in the peak amplitudes agrees with the results from the upwind and Godunov schemes studied by de Val-Borro et al. (2006). This correlation suggests that vortices can be formed by the non-linear evolution of Rossby waves in protoplanetary disks.

The evolution of the vortensity in the NIRVANA simulation with resolution $n_{\mathrm{r}} \times n_{\phi}=512 \times 1536$ is shown at several times in Fig. 10. The vortensity and Bernoulli constant are conserved for a barotropic inviscid fluid in the absence of discontinuities in the flow. In our case, the vortensity is roughly conserved along the streamlines in regions outside the Hill radius of the planet. Shock dissipation close to the protoplanet can lead to vortensity generation. As the gap is opened and strong trailing shocks are formed, the vortensity grows at the edge of the gap and along the spiral arms. The vortensity at 10 orbital periods shows small cavities outside the peaks at the edge of the gap. These depressions break in 4-5 differentiated vortices when the growth rate of the RWI becomes positive. The vortensity peak at the outer gap edge and close to the outer spiral arm are corrugated while at the inner edge the vortensity is more stable. As explained before, this is probably an artifi cial effect due to the inner edge of the gap being closer to the inner boundary than the outer gap edge is to the outer boundary. The minima of vortensity rotating along the edge correspond to vortices observed in the density maps. In the bottom right panel of Fig. 10 there is one single vortensity depression which is associated with a vortex located at azimuth $\approx \pi$ after 100 orbits. The vortensity inside the corotating region is considerably perturbed as the vortex moves along the gap.

In Figs. 11 and 12 the azimuthally averaged vortensity in the inertial frame is shown. The initial vortensity profile for a disk with uniform density, $\zeta_{0} \propto r^{-1.5}$, has been subtracted. The Cartesian FLASH code has a large vortensity excess in the corotating region where the gap is more depleted than in the cylindrical codes. The averaged vortensity in the outer disk is also greater in our Cartesian FLASH model. NIRVANA calculation shows vortensity peaks at the gap borders with a larger spike in the inner disk.

The velocity fi elds plotted over the density contours in logarithmic scale at $t=100$ orbits is shown in Fig. 13 . The velocity vectors are calculated in the corotating frame of the local maximum of pressure that coincides with the center of the vortex. 

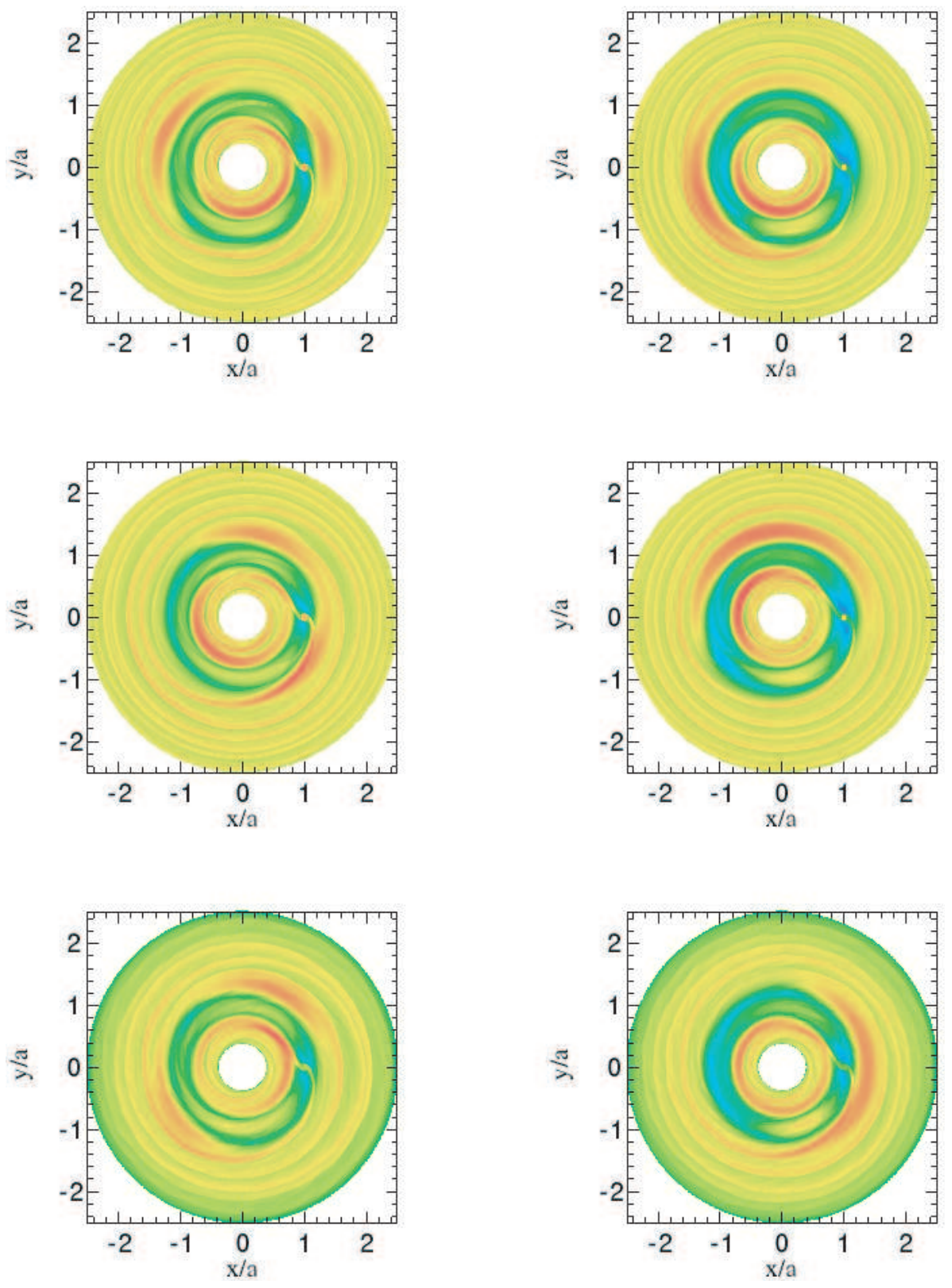

Fig. 6. Surface density contours for cylindrical simulations in logarithmic scale. From top to bottom, NIRVANA simulation using a damping wave region as described in Sect. 3 (see also de Val-Borro et al. 2006), NIRVANA simulation using outgoing-wave boundary conditions defined by Godon (1996) and polar FLASH. The left panels show the density after 50 orbital periods and the right panels show the density after 100 orbits with the same color scale. The resolution is $n_{r} \times n_{\phi}=256 \times 768$ and the planet is located at $x \times y=(1,0) a$. There are two elongated vortices forming next to the outer gap edge at 50 orbits that move with different velocities. The vortices merge in both simulations and one large vortex is observed in the outer disk at 100 orbits.

The rotation in baroclinic sense is very clearly visible in the vortex close to the inner edge of the gap. The vortex in the outer disk interacts with the spiral wake created by the planet and is perturbed at this particular time. In Fig. 14, the streamlines are shown in the frame corotating with the vortex core. They show baroclinic rotation that is perturbed by interaction with the spiral wakes created by the planet.

\subsection{Dependence on physical viscosity}

In this section we compare the unstable modes obtained from simulations including Navier-Stokes viscosity $v=10^{-6}$ and $10^{-5}$ (in code units, see Sect. 3).

In Fig. 15 we show the growth rates in the outer disk of the unstable modes with $m=5$ as a function of time for $v=10^{-6}$. The frequencies of unstable modes are calculated using perturbations on the inviscid Euler equations for simplicity. The growth rates at the outer edge of the gap agree within $20 \%$ with those obtained from inviscid simulations (see Fig. 5). However, notice that the linear analysis gives only an estimate of the growth rates in the viscous case.

\section{Discussion}

We have studied vortex formation in protoplanetary disks with an embedded giant planet, with mass ratios $10^{-4}$ and $10^{-3}$, using numerical simulations and linear perturbation analysis. The modal calculation is done following the strategy of Lovelace et al. (1999) for a locally isothermal equation of state in a vertically-averaged disk. Vortices are formed in the cylindrical NIRVANA and FLASH 2-dimensional simulations in agreement with the linear analysis of non-axisymmetric perturbations. The growth rates calculated for NIRVANA and polar FLASH as a function of time agree within about $10 \%$. The results of the linear analysis are consistent with the absence of rapidly growing vortices near the edge of the gap in our Cartesian-grid PPM simulations, which is thus not due to artifi cial numerical damping of 


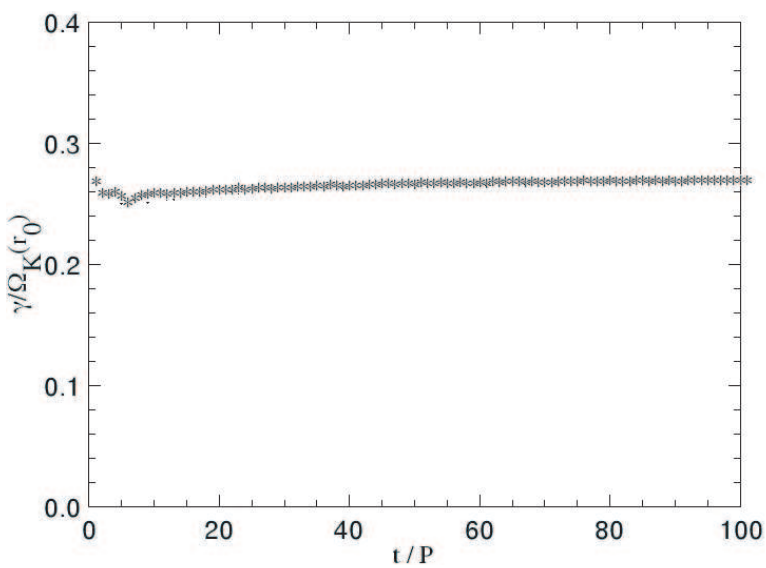

Fig. 7. Growth rates of the unstable modes with mode number $m=5$, as a function of time divided by the local Keplerian frequency. Dots represent a NIRVANA calculation with resolution $n_{r} \times n_{\phi}=256 \times 768$ and stars are obtained from a FLASH calculation with resolution $n_{r} \times n_{\phi}=$ $128 \times 384$

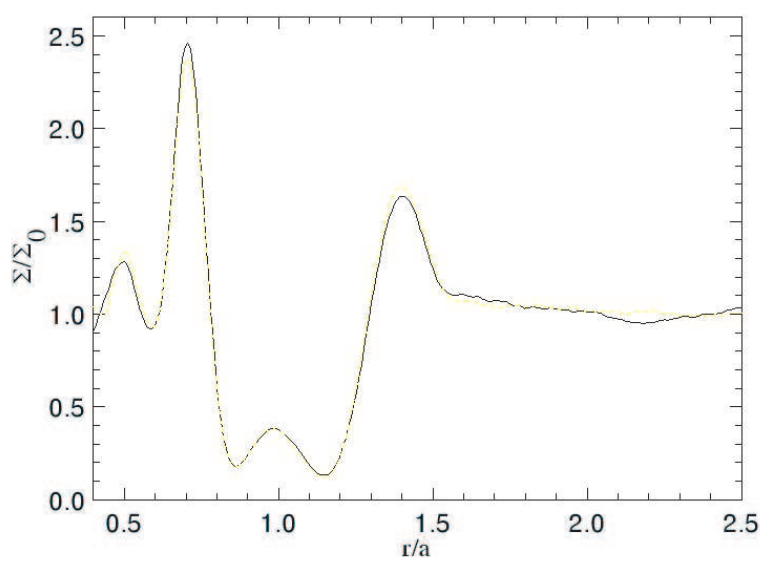

Fig. 8. Azimuthally averaged surface density profiles for the Jupiter simulations after 100 orbital periods. The solid line is the NIRVANA simulation with resolution $n_{r} \times n_{\phi}=256 \times 768$ and damping wave boundary conditions and the short dashed line is the NIRVANA simulation with resolution $n_{r} \times n_{\phi}=256 \times 768$ and outgoing-wave boundary conditions. The profiles are averaged over 5 orbits.

unstable modes. This type of code does not produce the necessary steepness of the surface density profi le and does not support growing non-axisymmetric perturbations.

Both our numerical schemes have very low numerical diffusion, which is estimated in Appendix A. Runs with an explicit Navier-Stokes viscosity were also performed. The unstable modes in the outer disk calculated for $v=10^{-6}$ have growth rates $\sim 0.25 \Omega_{\mathrm{K}}$ at 100 orbits, which are $20 \%$ smaller than in the inviscid calculations.

We speculate that the Cartesian-grid implementation may be more diffusive for this problem than polar geometry (in which the unperturbed Keplerian disk flows along the mesh structure) hence damping the growth of Rossby waves. The linear theory predicts that unstable modes will be present in the Cartesian simulations after the gap is sufficiently deep but these modes have smaller growth rate than those obtained from NIRVANA and FLASH polar simulations. The growth rate for the inner edge of the gap is larger than it is at the outer gap edge. This may be artifi cial because the inner boundary is closer to the planet position that the outer boundary is to the outer gap edge.
Table 1. The frequency in the corotating frame and magnitude of the maxima of the PDS of the gravitational torques from the inner disk are shown for the Jupiter simulations. The values are sorted by the magnitude of the PDS at the maximum. The magnitude of the PDS is related with the amplitude of the oscillations at a given frequency. Polar codes have larger maxima and the frequencies correspond roughly to the Keplerian frequencies at the gap edges.

\begin{tabular}{ccc}
\hline \hline Code & Frequency & Amplitude \\
\hline NIRVANA & 0.568 & $2.81 \times 10^{-5}$ \\
Flash polar & 0.531 & $8.96 \times 10^{-8}$ \\
Flash Cart. & 1.611 & $2.19 \times 10^{-7}$ \\
\hline
\end{tabular}

Table 2. The frequency in the corotating frame and magnitude of the maxima of the PDS of the gravitational torques from the outer disk are shown for the Jupiter simulations.

\begin{tabular}{ccc}
\hline \hline Code & Frequency & Amplitude \\
\hline NIRVANA & 0.389 & $1.11 \times 10^{-3}$ \\
Flash polar & 0.353 & $4.82 \times 10^{-6}$ \\
Flash Cart & 0.997 & $3.65 \times 10^{-6}$ \\
\hline
\end{tabular}

Table 3. The frequency and magnitude of the maxima of the PDS of the torques from the inner disk for the Neptune case are shown sorted by the magnitude of the PDS at the maximum. NIRVANA calculations have larger maxima with frequencies close to the Keplerian frequencies at the outer gap edge.

\begin{tabular}{ccc}
\hline \hline Code & Frequency & Amplitude \\
\hline NIRVANA & 0.204 & $4.67 \times 10^{-9}$ \\
Flash polar & 0.221 & $3.57 \times 10^{-8}$ \\
Flash Cart. & 0.345 & $3.99 \times 10^{-8}$ \\
\hline
\end{tabular}

Table 4. The frequency and magnitude of the maxima of the PDS of the torques from the outer disk for the Neptune case are shown sorted by the magnitude of the PDS at the maximum.

\begin{tabular}{ccc}
\hline \hline Code & Frequency & Amplitude \\
\hline NIRVANA & 0.389 & $1.11 \times 10^{-7}$ \\
Flash polar & 0.204 & $5.27 \times 10^{-8}$ \\
Flash Cart & 3.997 & $5.411 \times 10^{-7}$ \\
\hline
\end{tabular}

We observe a correlation between the growth rate of the unstable modes in the linear analysis and the size of the peaks in the power spectrum of the gravitational torque on the planet exerted by the disk. This correlation suggests that vortices in protoplanetary disks can form close to the gap, produced by an embedded giant planet, from the collapse of Rossby waves. Vortices may grow and be sustained for long timescales by interaction with the planetary wake (Koller et al. 2003; Li et al. 2005). The two-dimensional approximation for the disk flow is anticipated to give qualitatively correct results although a three-dimensional analysis is needed to understand heat dissipation in the vertical direction and refraction effects in radially propagating waves (Lin et al. 1990). An important restriction of our simulations is that the planet is kept on a fi xed circular orbit. It would be of interest to study how the vortices rotating along the edge of the gap affect the migration rate of a freely moving protoplanet embedded in a 3-dimensional disk.

In summary, the linear analysis confi rms that Rossby waves are formed in a thin protoplanetary disk with a giant planet with mass ratio between $\mu=10^{-4}-10^{-3}$, within tens of orbital 

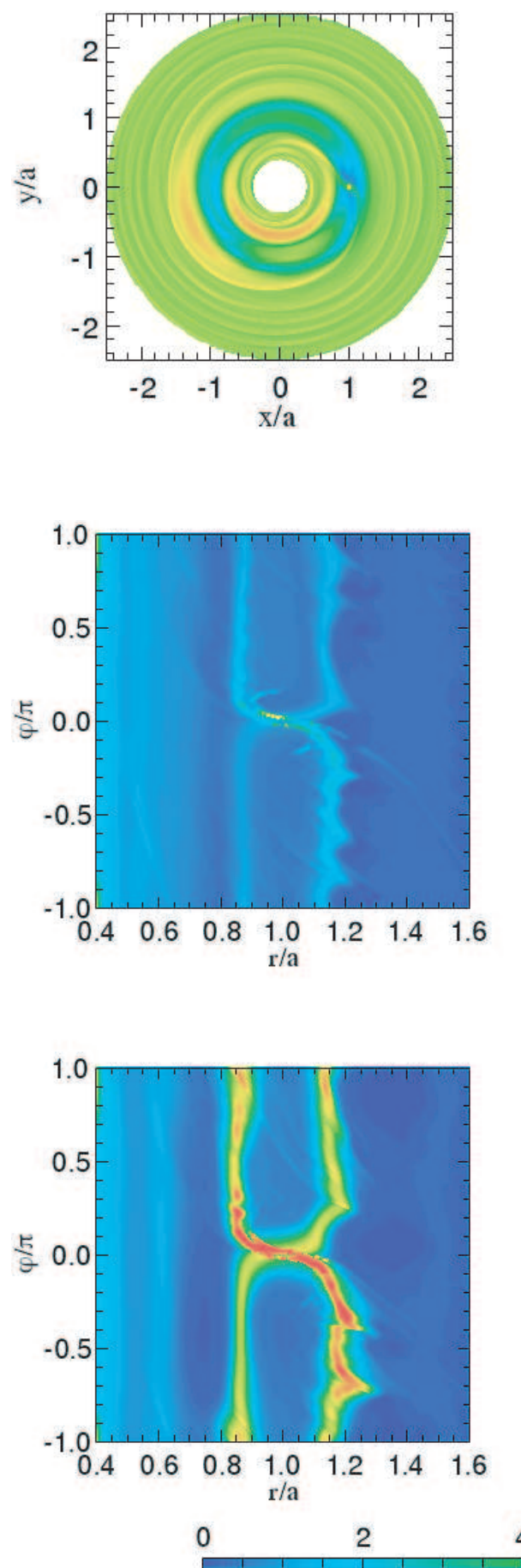
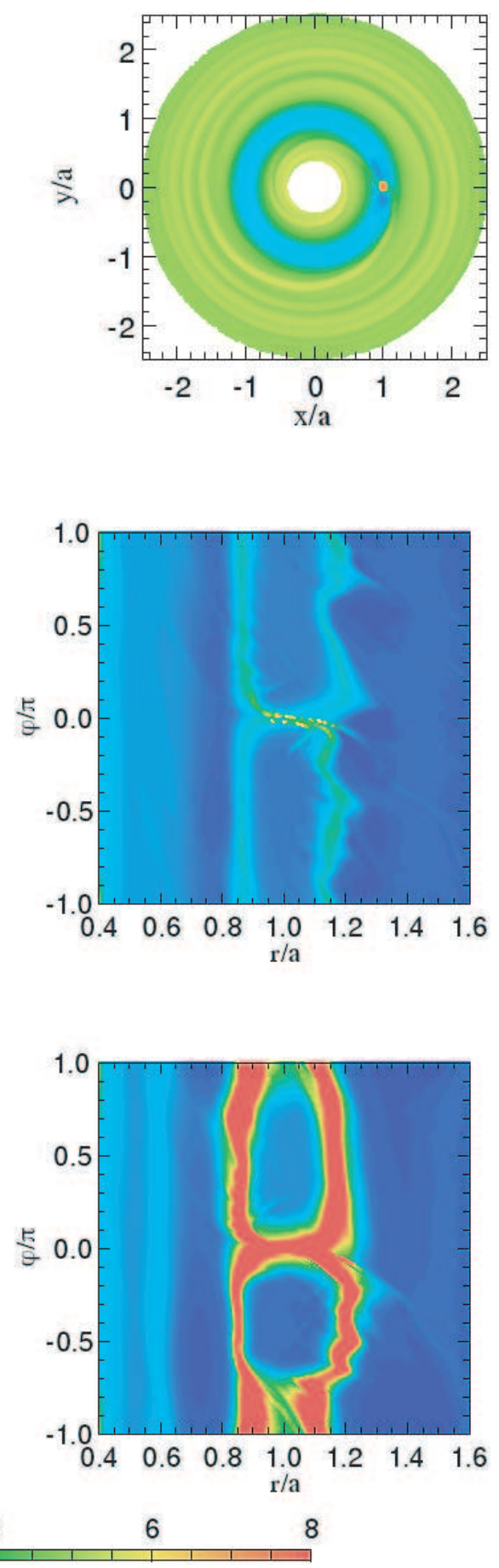

Fig. 9. Surface density distribution after 100 orbital periods for NIRVANA on the left hand side and FLASH on the right hand side using the same logarithmic color scale. Both models use the same wave damping condition in the outer disk between 2.1-2.5 $a$. while FLASH does not have a damping condition in the inner boundary. NIRVANA has density enhancements close to the gap opened by the protoplanet. FLASH has a smooth density distribution and a larger density peak at the planet position which is saturated in the image.
Fig. 10. Vortensity in polar coordinates is shown at times $t=10,20,50$ and 100 orbital periods from left to right and top to bottom. The simulation has resolution $n_{\mathrm{r}} \times n_{\phi}=512 \times 1536$. periods. The unstable modes with larger growth rates generate a non-axisymmetric perturbation, with mode number $m=4$ 6 , which breaks into vortices in the nonlinear regime producing a non-axisymmetric density distribution. At the time when the growth rate becomes positive, small depressions in vortensity appear along the gap. These results do not depend on resolution or boundary conditions. Simulations with an initial gap also generate vortices close to the edge of the gap. The growth rates estimated from the linear theory agree with the growth rates at later times in simulations with an initially flat density distribution. A protoplanetary disk with a giant planet becomes populated with vortices and spiral shocks that can efficiently transport angular momentum. This effect can be important in disks that are not sufficiently ionized to sustain turbulence via the MRI instability. We conclude that vorticity generation in protoplanetary disks with an embedded giant planet is a robust mechanism that can lead to planet formation and radial transfer of angular momentum.

Acknowledgements. M.d.V.B. was supported by a SAO predoctoral fellowship and a NOT/IAC scholarship. G.D. acknowledges support from the NASA Postdoctoral Program. We thank Artur Gawryszczak for providing his numerical code and enlightening discussions. The support of the RTN 'Planets" funded by the European Commission under agreement No. HPRN-CT-2002-0308 is acknowledged during the course of this project. The FLASH code used in this work is developed in part by the US Department of Energy under Grant 


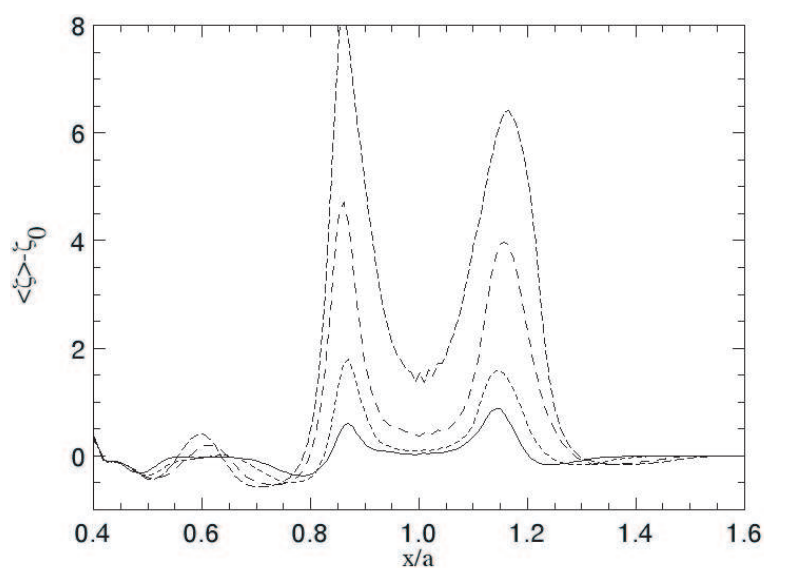

Fig. 11. Vortensity profiles averaged over azimuth at different times $t=10,20,50$ and 100 orbital periods for the NIRVANA simulation at resolution $n_{\mathrm{r}} \times n_{\phi}=256 \times 768$.

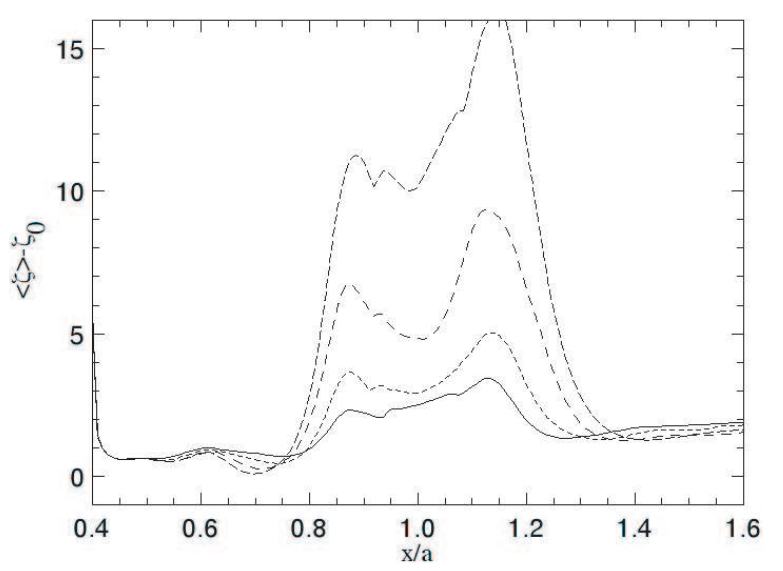

Fig. 12. Vortensity profiles averaged over azimuth at different times $t=10,20,50$ and 100 orbital periods for the FLASH simulation in Cartesian coordinates.

No. B523820 to the Center for Astrophysical Thermonuclear Flashes at the University of Chicago. Some of the calculations reported here were performed on Columbia, operated by NASA Advanced Supercomputing Division, at NASA Ames Research Center. We thank the anonymous referee for comments that improved the manuscript.

\section{Appendix A: Calibration of numerical viscosity}

The calculations presented in this paper are based on inviscid Euler equations. However, even though neither physical nor artifi cial viscosity terms enter these equations, each numerical scheme has some intrinsic diffusivity that can be interpreted as a numerical viscosity, $v_{\mathrm{n}}$.

To calibrate the numerical viscosity in each of the hydrodynamics codes, we use the numerical setup described in Sect. 3.1 with mass ratio $q=0$. Tests are performed at two different resolutions to check for consistency of the results. Numerical viscosity is bound to depend on the flow properties. The values reported in this Appendix apply to disks in quiescent conditions and may therefore represent lower limits for numerical diffusion in models with uniform initial density and fast gap formation.

In the case of NIRVANA, a time-averaged measure of the numerical diffusivity is obtained over a 50 orbit period, as a function of the radial position, by analysing the trajectories of
500 tracer (massless) particles released in the disk. The equation of motion of each particle is integrated every hydrodynamics timestep by interpolating the velocity fi eld at the particle's location and advancing in time its position by means of a secondorder Runge-Kutta method. The spatial interpolation of the velocity fi eld is also second-order accurate. Hence, trajectories are formally second-order accurate in both space and time.

We assume that the viscosity is constant in a radial interval $\Delta r=0.1$, which contains about 25 equally-spaced tracer particles and is orders of magnitude larger than their diffusion length scale. We measure the averaged length travelled by the particles in each radial interval, over about 50 orbits (at $r=1$ ), and estimate the amount of numerical viscosity under the assumption that the particle drift velocity is

$\left|\frac{\mathrm{d} r}{\mathrm{~d} t}\right|=\frac{3 v_{\mathrm{n}}}{2 r}$

Experiments executed at resolutions $n_{\mathrm{r}} \times n_{\phi}=256 \times 768$ and $512 \times 1536$ provide very similar results.

The largest numerical diffusion is observed close to the inner grid boundary, where $v_{\mathrm{n}} \sim 10^{-7}$ (in code units, see Sect. 3.1) at $r \approx 0.55$ and $\sim 10^{-8}$ at $r \approx 0.65$. In the radial domain between $r \approx 0.75$ and $r \sim 1.75, v_{\mathrm{n}}$ lies between $\sim 10^{-10}$ and $\sim 10^{-9}$. In the outer part of the simulated disk, the numerical viscosity is comprised between $\sim 10^{-9}$ and $\sim 10^{-8}$.

The numerical viscosity in FLASH is calibrated using a 2-dimensional local patch of a Keplerian disk with a massless sink hole in the center of the domain The sink hole has radius equal to the Roche radius

$R_{\mathrm{R}}=a\left(\frac{q}{3}\right)^{1 / 3}$

for a protoplanet with mass ratio $q=10^{-3}$. The dimensions of the shearing box are $20 R_{\mathrm{R}} \times 20 R_{\mathrm{R}}$. We use local Cartesian coordinates in the Hill approximation corotating with the sink hole. The horizontal axis corresponds to the radial direction and the vertical axis to the direction of motion of the flow. Initially, the unperturbed surface density is uniform and the velocity of the matter flowing into the computational domain has only a vertical component given by the linearized Keplerian velocity

$v_{y}=-\frac{3}{2} \Omega_{\mathrm{K}} x$.

We implement periodic boundaries in the vertical direction. Our units in the simulation are the Roche radius, $R_{\mathrm{R}}$, the initial surface density, $\Sigma_{0}$, and the Keplerian angular frequency, $\Omega_{\mathrm{K}}$, at the center of the shearing box. A cavity is produced at the orbital radius of the sink hole while the gas in the vicinity of the hole is pulled into it on the viscous timescale.

The equation of viscous diffusion for a thin accretion disk can be obtained in the asymptotic limit assuming constant numerical diffusivity (Pringle 1981; Bryden et al. 1999). Using the boundary conditions $\Sigma=0$ at $x=0$, and $\partial \Sigma / \partial x=0$ at $x=x_{\text {out }}$, where $x_{\text {out }}=10 R_{\mathrm{R}}$ is the distance from the center of the shearing box to the outer radial boundary, the surface density distribution for positive $x$ is given by

$\Sigma(x, t)=\Sigma_{0} \exp \left(-\frac{3 \pi^{2} v_{\mathrm{n}} t}{4 x_{\text {out }}^{2}}\right) \sin \left(\frac{\pi x}{2 x_{\text {out }}}\right)$.

The kinematic viscosity can be evaluated from the ratio of surface densities at different times

$v_{\mathrm{n}}=\frac{4 x_{\mathrm{out}}^{2}}{3 \pi^{2}\left(t_{2}-t_{1}\right)} \ln \left[\frac{\Sigma\left(t_{1}\right)}{\Sigma\left(t_{2}\right)}\right]$. 

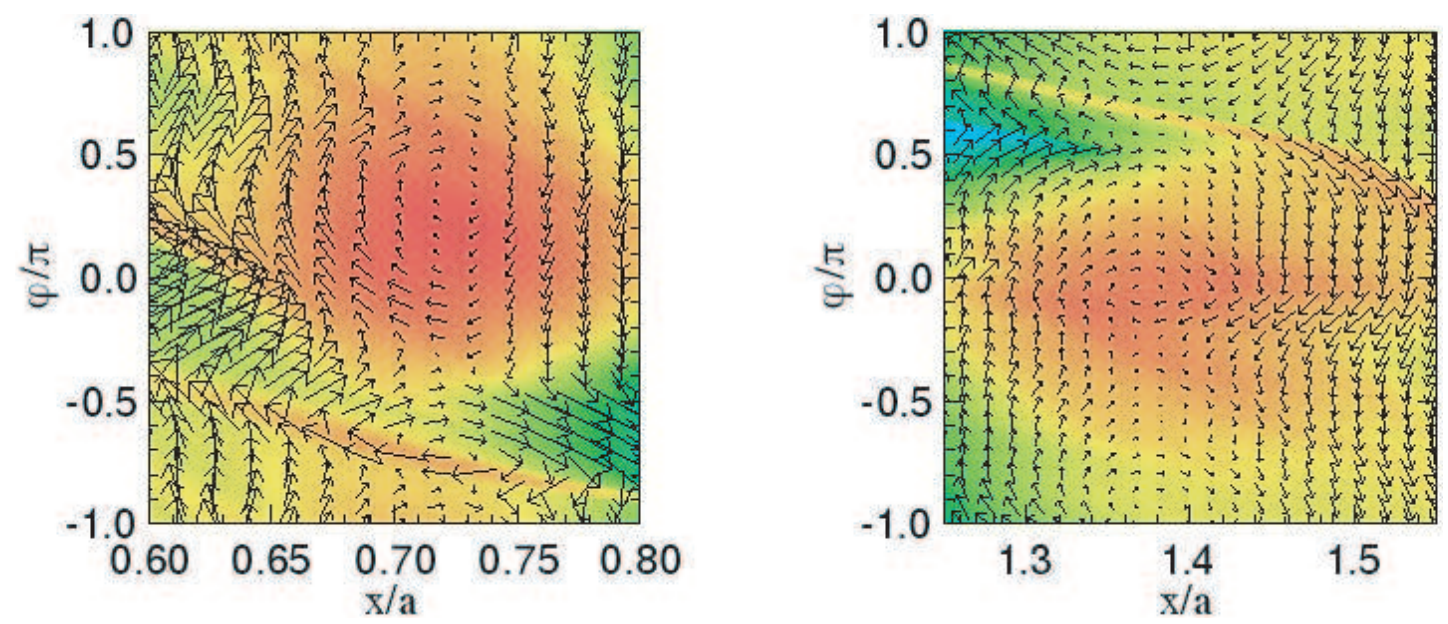

Fig. 13. Velocity vectors in the corotating frame after 100 periods for the NIRVANA simulation at resolution $n_{\mathrm{r}} \times n_{\phi}=512 \times 1536$. The left panel shows the vortex at the inner gap edge and the right panel shows the vortex at the outer gap edge.
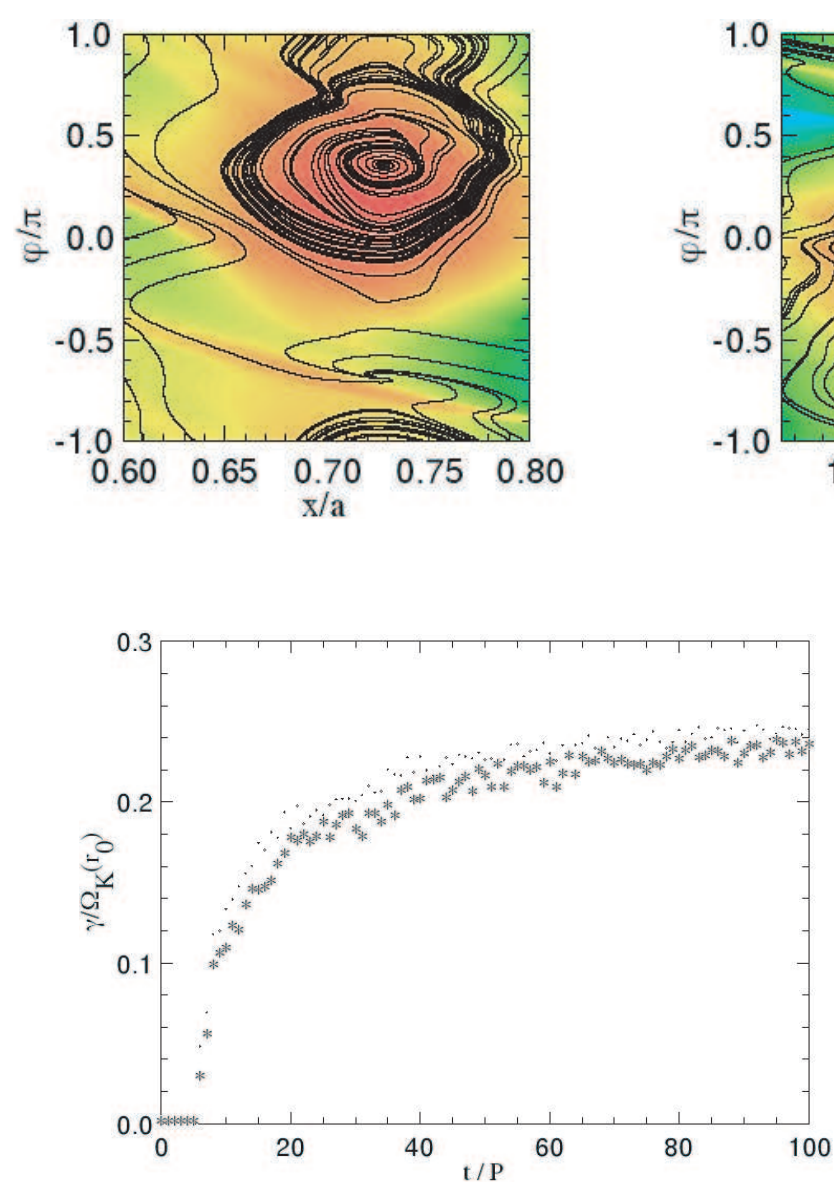

Fig. 15. Dependence on time of growth rates of modes with $m=5$ for NIRVANA simulations with resolution $n_{\mathrm{r}} \times n_{\phi}=256 \times 768(\mathrm{dots})$ and FLASH with resolution $n_{\mathrm{r}} \times n_{\phi}=128 \times 384$ (stars). The Navier-Stokes viscosity is $v=10^{-6}$.

We estimate values of $v_{\mathrm{n}}$ between $10^{-8}$ and $10^{-7}$ using the surface density profi les averaged in the vertical direction at times close to 50 orbits and grid resolutions of $n_{x} \times n_{y}=320 \times 320$ and $640 \times 640$.

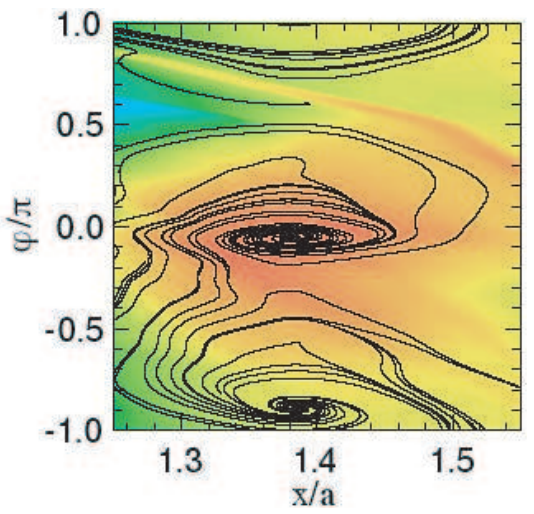

Fig. 14. Streamlines in the corotating frame of the inner and outer vortices plotted in Fig. 13. The radial extent of the vortices is about $0.15 a$. Spiral arms created by the planet and weaker shocks associated with the vortices are observed.

\section{References}

Balbus, S. A. 2003, ARA\&A, 41, 555

Balbus, S. A., \& Hawley, J. F. 1991, ApJ, 376, 214

Balbus, S. A., \& Hawley, J. F. 1998, Rev. Mod. Phys., 70, 1

Balbus, S. A., \& Hawley, J. F. 2006, ApJ, 652, 1020

Balbus, S. A., Hawley, J. F., \& Stone, J. M. 1996, ApJ, 467, 76

Balmforth, N. J., \& Korycansky, D. G. 2001, MNRAS, 326, 833

Barge, P., \& Sommeria, J. 1995, A\&A, 295, L1

Barranco, J. A., \& Marcus, P. S. 2005, ApJ, 623, 1157

Barranco, J. A., \& Marcus, P. S. 2006, J. Comp. Phys., 219, 21

Bracco, A., Chavanis, P. H., Provenzale, A., \& Spiegel, E. A. 1999, Phys. Fluids, 11,2280

Bryden, G., Chen, X., Lin, D. N. C., Nelson, R. P., \& Papaloizou, J. C. B. 1999, ApJ, 514, 344

Calder, A. C., Fryxell, B., Plewa, T., et al. 2002, ApJS, 143, 201

Ciecielag, P., Plewa, T., \& Różyczka, M. 2000, Astron. Nachr., 321, 171

Colella, P., \& Woodward, P. 1984, J. Comput. Phys., 54, 174

Colgate, S. A., Cen, R., Li, H., Currier, N., \& Warren, M. S. 2003, ApJ, 598, L7

de Val Borro, M., \& Artymowicz, P. 2004, in Extrasolar Planets: Today and

Tomorrow, ed. J. Beaulieu, A. Lecavelier Des Etangs, \& C. Terquem, ASP

Conf. Ser., 321, 333

de Val-Borro, M., Edgar, R. G., Artymowicz, P., et al. 2006, MNRAS, 370, 529

Dubrulle, B., Marié, L., Normand, C., et al. 2005, A\&A, 429, 1

Fryxell, B., Olson, K., Ricker, P., et al. 2000, ApJS, 131, 273

Gammie, C. F. 1996, ApJ, 457, 355

Godon, P. 1996, MNRAS, 282, 1107

Goldreich, P., Goodman, J., \& Narayan, R. 1986, MNRAS, 221, 339

Ji, H., Burin, M., Schartman, E., \& Goodman, J. 2006, Nature, 444, 343

Johnson, B. M., \& Gammie, C. F. 2006, ApJ, 636, 63

Kargl, S. G., \& Marston, P. L. 1989, Acoust. Soc. Am. J., 85, 1014

Klahr, H. H., \& Bodenheimer, P. 2003, ApJ, 582, 869

Klahr, H., \& Bodenheimer, P. 2006, ApJ, 639, 432 
Kley, W. 1998, A\&A, 338, L37

Koller, J., Li, H., \& Lin, D. N. C. 2003, ApJ, 596, L91

Laughlin, G., Korchagin, V., \& Adams, F. C. 1998, ApJ, 504, 945

Li, H., Finn, J. M., Lovelace, R. V. E., \& Colgate, S. A. 2000, ApJ, 533, 1023

Li, H., Colgate, S. A., Wendroff, B., \& Liska, R. 2001, ApJ, 551, 874

Li, H., Li, S., Koller, J., et al. 2005, ApJ, 624, 1003

Lin, D. N. C., \& Papaloizou, J. 1985, in Protostars and Planets II, ed. D. C. Black, \& M. S. Matthews, 981

Lin, D. N. C., Papaloizou, J. C. B., \& Savonije, G. J. 1990, ApJ, 364, 326 Lovelace, R. V. E., Li, H., Colgate, S. A., \& Nelson, A. F. 1999, ApJ, 513, 805 Lubow, S. H., \& D’Angelo, G. 2006, ApJ, 641, 526

Lubow, S. H., Seibert, M., \& Artymowicz, P. 1999, ApJ, 526, 1001

Marcus, P. S. 1988, Nature, 331, 693

Mukhopadhyay, B., Afshordi, N., \& Narayan, R. 2005, ApJ, 629, 383

Nelson, A. F., \& Benz, W. 2003, ApJ, 589, 556

Papaloizou, J. C. B., \& Pringle, J. E. 1984, MNRAS, 208, 721

Papaloizou, J. C. B., \& Pringle, J. E. 1985, MNRAS, 213, 799
Papaloizou, J. C. B., \& Pringle, J. E. 1987, MNRAS, 225, 267

Plewa, T., \& Müller, E. 2001, Comp. Phys. Comm., 138, 101

Press, W. H., Teukolsky, S. A., Vetterling, W. T., \& Flannery, B. P. 1992,

Numerical recipes in FORTRAN. The art of scientifi c computing, 2nd edn. (Cambridge: University Press)

Pringle, J. E. 1981, ARA\&A, 19, 137

Richard, D. 2001, Ph.D. Thesis, AA (Observatoire de Paris GIT/SPEC CEA Saclay)

Richard, D., \& Zahn, J.-P. 1999, A\&A, 347, 734

Shakura, N. I., \& Sunyaev, R. A. 1973, A\&A, 24, 337

Tagger, M. 2001, A\&A, 380, 750

Tagger, M., \& Melia, F. 2006, ApJ, 636, L33

Varnière, P., \& Tagger, M. 2006, A\&A, 446, L13

Weirs, G., Dwarkadas, V., Plewa, T., Tomkins, C., \& Marr-Lyon, M. 2005, Ap\&SS, 298, 341

Woodward, P., \& Colella, P. 1984, J. Comput. Phys., 54, 115

Ziegler, U., \& Yorke, H. W. 1997, Comp. Phys. Comm., 101, 54 